Association for Information Systems

AIS Electronic Library (AISeL)

Wirtschaftsinformatik Proceedings 2001

Wirtschaftsinformatik

September 2001

\title{
Analyse der ERP-Outsourcingentscheidung im Spannungsfeld von technologischem und unternehmensbezogenem Wissen
}

Lars Brehm

Universität Bayreuth, Lars.Brehm@uni-bayreuth.de

Jens Dibbern

Universität Bayreuth, jens.dibbern@uni-bayreuth.de

Follow this and additional works at: http://aisel.aisnet.org/wi2001

\section{Recommended Citation}

Brehm, Lars and Dibbern, Jens, "Analyse der ERP-Outsourcingentscheidung im Spannungsfeld von technologischem und unternehmensbezogenem Wissen" (2001). Wirtschaftsinformatik Proceedings 2001. 51.

http://aisel.aisnet.org/wi2001/51

This material is brought to you by the Wirtschaftsinformatik at AIS Electronic Library (AISeL). It has been accepted for inclusion in Wirtschaftsinformatik Proceedings 2001 by an authorized administrator of AIS Electronic Library (AISeL). For more information, please contact elibrary@aisnet.org. 
In: Buhl, Hans Ulrich, u.a. (Hg.) 2001. Information Age Economy; 5. Internationale Tagung Wirtschaftsinformatik 2001. Heidelberg: Physica-Verlag

ISBN: 3-7908-1427-X

(C) Physica-Verlag Heidelberg 2001 


\title{
Analyse der ERP-Outsourcingentscheidung im Spannungsfeld von technologischem und unternehmensbezogenem Wissen
}

\author{
Lars Brehm, Jens Dibbern ${ }^{1}$ \\ Universität Bayreuth
}

Zusammenfassung: Im letzten Jahrzehnt haben ERP-Systeme eine hohe Verbreitung in der Wirtschaft erfahren. Oftmals wird ihnen die Rolle einer "mission critical"-Anwendung für die Aufrechterhaltung des Geschäftsbetriebs zugeschrieben. Innerhalb der Phasen der Auswahl, Implementierung und Stabilisierung sowie dem Betrieb und der Verbesserung des ERP-Systems greifen viele Unternehmen auf die Unterstützung externer Dienstleister zurück. Manche Unternehmen lagern im Zuge der Entscheidung für ein ERP-Paket sogar ihre gesamte interne Informationsverarbeitung aus. In dem Beitrag wird analysiert, wie verschiedene Wissenskategorien die Entscheidung über das Outsourcing wesentlicher Aufgaben, die im Rahmen des ERP-Lebenszyklus anfallen, beeinflussen. Auf Basis der Erkenntnisse aus der bisherigen Literatur zum Outsourcing der Informationsverarbeitung werden die wesentlichen wissensbezogenen Entscheidungsdeterminanten des Outsourcing der Informationsverarbeitung herausgearbeitet und in einem Strukturmodell zusammengefasst. Es zeigt sich, dass insbesondere die Beurteilung der internen Ressourcen und Fähigkeiten gegenüber dem Markt und die Spezifität des technologischen Wissens und des Unternehmenswissens die Outsourcingentscheidung beeinflussen. In einem zweiten Schritt wird ein Bezugsrahmen entwickelt, mit dessen Hilfe die einzelnen Aufgaben des ERP-Lebenszyklus verschiedenen Wissenskategorien sowie internen und externen Aufgabenträgern zugeordnet werden können. Anhand eines Beispiels wird illustriert, wie die in der Literatur ermittelten Entscheidungsdeterminanten des Outsourcing der Informationsverarbeitung als Kriterien der Zuordnung angewendet werden können.

Schlüsselworte: Outsourcing, Wissen, Resource-based Theory, Transaktionskostentheorie, ERP-Pakete, ERP-Systeme, ERP-Lebenszyklus, Risikopotenziale

\footnotetext{
Die Autoren danken Prof. Dr. Armin Heinzl für die wertvollen Hinweise zur Verfeinerung des Beitrags.
} 


\section{Einleitung}

Sowohl der Markt für ERP-Systeme als auch der Markt für externe IT-Dienstleistungen ist in den letzten zehn Jahren durch stetige Wachstumsraten gekennzeichnet. Beide Marktentwicklungen bedingen sich gegenseitig. ERP-Softwarepakete werden nach den allgemeinen Anforderungen einer Klasse von Unternehmen (z.B. Branche, Fertigungsart, etc.) und nicht nach den spezifischen Anforderungen eines einzelnen Unternehmens entwickelt. Die damit verbundene Standardisierung fördert die Entstehung von Softwaremärkten und zieht die Entstehung von Dienstleistungsmärkten nach sich.

Bevor ein Unternehmen ein ERP-Paket nutzen kann, muß das Softwarepaket erst entsprechend der unternehmensindividuellen organisatorischen Prozesse und Strukturen konfiguriert werden. Die Konfiguration beinhaltet das Setzen von Parametern (bei SAP R/3 über 5.000) innerhalb der Grenzen der vom ERP-Hersteller zur Verfügung gestellten Funktionalität [ApRi00]. Es können sich allerdings Konfigurationsprobleme ergeben, wenn die Funktionalität des ERP-Pakets nicht den Erfordernissen der Geschäftsprozesse des Unternehmens entspricht. In diesem Fall gibt es drei Möglichkeiten, um eine Lösung herbeizuführen. Erstens können die Unternehmensprozesse an die Software angepasst werden. Zweitens besteht die Option, den Quellcode des ERP-Pakets zu ändern oder zu ergänzen. In diesem Zusammenhang wird von Modifikationen gesprochen [Krcm97; MaTa00]. Drittens gibt es die Möglichkeit, eine systemfremde Softwarelösung (Satelitensysteme, z.B. Legacy Systems) an das ERP-System anzubinden [Krcm97]. Sowohl die Schnittstellenprogrammierung als auch die Modifikation können bei einem späteren Releasewechsel zu erneuten Anpassungsproblemen führen. Auch werden durch Abweichungen vom Standard die integrativen Vorteile des ERP-Systems eingeschränkt [MaTa00]. ${ }^{2}$

Alle diese Tätigkeiten verlangen sowohl Wissen über die produkt- und funktionsbezogenen Eigenschaften der ERP-Software als auch über die Bedürfnisse der Unternehmung. Beide Wissensarten sind bei der Auswahl des richtigen ERP-Pakets sowie bei der Implementierung und der nachgelagerten Stabilisierungsphase und dem Betrieb des ERP-Systems (Phasen des ERP-Lebenszyklus) in unterschiedlichem Maße relevant. Externe Dienstleister haben erkannt, dass sie beim Aufbau des Softwarewissens Spezialisierungsvorteile gegenüber unternehmensinternen Abteilungen der Informationsverarbeitung (IV) realisieren können. Zum heutigen Zeitpunkt existieren eine Vielzahl von ERP-Dienstleistern, z.B. in Form von Systemhäusern oder Beratungsunternehmen, die teilweise als Kooperationspartner der Hersteller fungieren. Während sie die ERP-Systeme kennen und produkt- und funktionsbezogene Spezialisierungsvorteile aufweisen, verfügen die

2 [BrHM01] zeigen auf, dass noch weitere Möglichkeiten der Anpassung von ERPPaketen existieren. Auf diese wird aber im Weiteren aus Gründen der Vereinfachung nicht eingegangen. 
Kunden über das unternehmensspezifische Wissen [Hein96, S. 285f.]. Aufgrund dieser Wissensasymmetrie haben sich vielfältige Formen der zwischenbetrieblichen Arbeitsteilung bei der Verrichtung der Aufgaben, die innerhalb der einzelnen Phasen des ERP-Lebenszyklus anfallen, herausgebildet. Im Folgenden sollen diese verschiedenen Formen der Inanspruchnahme des Dienstleistungsmarkts als Varianten des IV-Outsourcing verstanden werden.

Der Begriff des IV-Outsourcing läßt sich anhand der Dimensionen Umfang, Dauer und Eigentumsverhältnisse definieren. Der Umfang des IV-Outsourcing wird sowohl durch den Gegenstand als auch durch den Grad des IV-Outsourcing festgelegt. In der Literatur werden verschiedene Funktionen der IV unterschieden: z.B. Anwendungsentwicklung und Anpassung von Standardsoftware; Wartung und Betreuung von Anwendungssystemen und Hardware; Systembetrieb / Rechenzentrum; Aufbau, Betrieb und Wartung von Netzwerken, Telekommunikationseinrichtung; Anwenderunterstützung und -schulung sowie Planung und Management [MeKn98]. Zur Verrichtung der Aufgaben der einzelnen Funktionen sind in unterschiedlichem Maße physische und menschliche Ressourcen vonnöten. Diese können entweder partiell oder vollständig von einem externen Dienstleister übernommen und/oder von diesem zur Verfügung gestellt werden. Verbleiben nur die physischen Ressourcen im Eigentum des Kunden, so spricht man auch von Facilities Management. Eine weitere auf besondere Eigentumsverhältnisse zurückzuführende Spezialform des Outsourcing beinhaltet den Übergang von Funktionen der IV an ein hundertprozentiges Tochterunternehmen. In diesem Fall wird von einer Ausgliederung gesprochen [Hein93]. Bei gegenseitiger Kapitalbeteiligung liegt ein Joint-Venture vor. Die Vertragsbeziehungen mit dem externen Dienstleister können langfristig oder kurzfristig (z.B. projektbezogen oder auf Stundenbasis) angelegt sein.

Grundsätzlich gilt, dass die Einführung eines ERP-Systems Veränderungen in allen Funktionen der IV herbeiführen kann. Bei jeder dieser Neuerungen stellt sich potenziell die Frage, ob die Ressourcen und Fähigkeiten ausreichen, um die einzelnen Aufgaben intern durchzuführen. Werden Wissensdefizite festgestellt, so ergibt sich die Frage, welche Risiken sich mit einer Auslagerung dieser Aufgaben ergeben und ob diese durch die Gestaltung der Dauer, des Umfangs und der Eigentumsverhältnisse, sowie durch vertragliche Regelungen oder durch das Beziehungsmanagement abgeschwächt werden können.

In dem vorliegenden Beitrag soll die Outsourcingentscheidung unter besonderer Berücksichtigung des technologischen und unternehmensbezogenen Wissens, das in den einzelnen Aufgaben des ERP-Lebenszyklus notwendig ist, analysiert werden. 


\section{Die Bedeutung des Wissens beim IV-Outsourcing}

Der Aspekt des Wissens fand in einer Vielzahl von empirischen Studien Berücksichtigung, die nach den Determinanten des Outsourcing der Informationsverarbeitung fragen. Die Mehrzahl der Arbeiten ergründen, unter welchen notwendigen und hinreichenden Bedingungen (Varianztheorie [Mohr82, S. 37ff.]), Unternehmen Funktionen der Informationsverarbeitung an externe Dienstleister auslagern.

Es lassen sich zwei wesentliche Begründungszusammenhänge der Outsourcingentscheidung unterscheiden [PiMa92, DiGH01]:

1. Die Unternehmen treffen einen Vergleich der Produktions- und Transaktionskosten interner versus externer Erstellung. Die Kostenunterschiede sind abhängig von den Charakteristika der IV-Funktionen (Transaktionskostentheorie [Will81]).

2. Sie beurteilen die eigenen Ressourcen und Fähigkeiten in der IV gegenüber dem Markt unter Berücksichtigung ihres strategischen Potenzials. Bei eigenen Defiziten kommt die Inanspruchnahme des Markts im Sinne von komplementären Ressourcen und Fähigkeiten in Betracht [TRDW94] (Resource-based Theory [Barn91]).

\subsection{Transaktionskostentheorie}

Im Rahmen der Transaktionskostentheorie ist der Wissensaspekt mit der Faktorspezifität verbunden. Es kann zwischen technologischer Spezifität und Spezifität des Humankapitals unterschieden werden. Grundsätzlich führt eine hohe Faktorspezifität dazu, dass ein externer Dienstleister spezifische Investitionen tätigen muss. Basierend auf der Annahme, dass sich der Anbieter stets opportunistisch verhält, wird er versuchen, diese Investitionen während der Vertragsdauer zu überkompensieren. Um dem entgegenzuwirken, fallen erhebliche Kosten der Vertragsanbahnung, -gestaltung, und -kontrolle an (Transaktionskosten). Zudem wird der Externe in diesem Fall keine Produktionskostenvorteile im Sinne von Skalenerträgen realisieren können, so dass ein Kostenvergleich zu Gunsten der internen Erstellung ausfällt.

In den gesichteten Arbeiten mit konfirmatorischem Charakter hat sich dieser $\mathrm{Zu}$ sammenhang in Abhängigkeit von der Art der Operationalisierung der Faktorspezifität bestätigt. Bei Konstrukten, in denen primär auf die Spezifität der Hardwareund Softwarekomponenten [NRRC96, Loh94] oder auf die funktionale Komplexität [AnCu97] abgestellt wurde, konnte kein Einfluss auf den Grad der Auslagerung festgestellt werden. Bei einer Operationalisierung im Sinne von implizitem technologischem Wissen [NRRC96], technologischem und geschäftsprozessspezifischem Wissen [AnCu97] oder der Notwendigkeit von Unternehmenswissen, unternehmensindividueller Funktionalität und hohen Kosten eines Anbieterwech- 
sels [PoZe98] konnte hingegen ein negativer Einfluss auf den Grad der Auslagerung von IV-Funktionen nachgewiesen werden. In der bislang einzigen Untersuchung in kleinen und mittleren Unternehmen (KMU) konnte kein Zusammenhang zwischen der Faktorspezifität des Humankapitals in IV-Funktionen (Notwendigkeit von Kenntnissen über Arbeitsabläufe in und zwischen Fachabteilungen sowie bereichsübergreifende Team- und Projektarbeit) und deren Auslagerungsgrad festgestellt werden [DiHe01].

\subsection{Resource-based Theory}

Bestätigt hat sich in dieser Studie [DiHe01] allerdings die Hypothese, dass KMU bei Ressourcendefiziten gegenüber dem Markt - in Form von gegenwärtigen Leistungs- und zukünftigen Wissensdefiziten - vermehrt Funktionen der IV auslagern. Dieser Zusammenhang basiert auf der Resource-based Theory und konnte in der Studie von [TeCG95] auch bezogen auf Defizite in der Personal- und Unterstuitzungsqualität der IV nachgewiesen werden. Darüber hinaus gibt es eine Reihe von Studien, die sich zwar nicht explizit auf die Resource-based Theory beziehen, aber ähnliche Argumentationen anführen. [Loh94] findet Bestätigung für die Hypothese, dass mit dem IV-Outsourcing Vorteile durch den Zugang zu neuen Technologien, Expertenwissen und der Promotion von innovativem Gebrauch der IV assoziiert werden (technologischer Imperativ). Die Folge ist ein erhöhter Grad der IV-Auslagerung. Die Ergebnisse der Arbeit von [PoZe98] bestätigen, dass Unternehmen Funktionen der IV umso mehr auslagern, je umfassender die Anforderungen an die benötigten Fähigkeiten ausfallen. [NeRS96] können nachweisen, dass die Entwicklung von Anwendungssystemen, die auf spezifischen Technologien und fortgeschrittenen Entwicklungsumgebungen beruhen, eher ausgelagert werden oder mit dem Zugriff auf eine Standardsoftware verbunden sind. Sie können keine Unterschiede zwischen dem Einfluss des Umfangs der Anwendungen und den technologischen Eigenschaften auf die Outsourcingentscheidung feststellen. [NRRC96] zeigen auf, dass eine starke Systemheterogenität mit einem erhöhten Grad der Auslagerung von IV-Funktionen verbunden ist.

Die Tatsache, dass Unternehmen Funktionen der IV aufgrund eigener Wissensdefizite außer Haus geben, wird von der Literatur allerdings auch kritisch reflektiert. [LaWF95, S. 91] warnen auf Basis ihrer umfangreichen Fallstudienuntersuchung vor dieser Praxis. Während sie primär auf das Risiko hoher nachvertraglicher Kosten verweisen, die auf opportunistische Verhaltensweisen des externen Anbieters zurückzuführen sind, betont [Ear196] in seiner konzeptionellen Arbeit das Risiko des Verlusts innovativer Fähigkeiten im Unternehmen. [Dunc98] führt an, dass externe Dienstleister oftmals starkes technologisches Wissen hinsichtlich der Spezifizierung und Funktionalität von Informationssystemen aufweisen. Ihnen fehle aber das Unternehmenswissen, das notwendig sei, um die Potenziale der Technologie voll auszuschöpfen. Gleichzeitig sei innerhalb der Unternehmung technologisches Wissen wichtig, um ihr wertsteigerndes Potenzial in der Zukunft zu er- 
kennen. Das kurzfristige Schließen von Wissenslücken mit Hilfe eines externen Anbieters berge demnach die Gefahr der langfristigen Erosion von Wissen, Kontrolle und Flexibilität im Erkennen der Potenziale der IV. Dieses geht über die Gefahr des aus der Transaktionskostentheorie bekannten opportunistischen Verhaltens hinaus. Im Gegensatz dazu argumentieren [QuHi94], dass der Zugang zu überlegenen technologischen Ressourcen nicht auf den internen Innovationsprozess beschränkt sei. Zwar leugnen sie nicht, dass Outsourcing strategische Risiken wie den Verlust von technologischen Fähigkeiten und entsprechender Kontrollmöglichkeiten berge. Sie führen aber an, dass dieses Risiko durch entsprechende vertragliche Beziehungen abgeschwächt werden könne.

\subsection{Ausdrückliche Berücksichtigung des Wissens}

[Scar98] leistet einen Beitrag zur Verminderung der dargestellten Kontroverse, indem er die Auslagerung von IV-Funktionen ausdrücklich als alternative Form der Organisation von Wissen begreift. Neben den bereits erläuterten Transaktionskosten und dem Innovationspotenzial verschiedener Arten des Wissens führt er als Abgrenzungskriterium die Vermittelbarkeit von Wissen ein. Für ihn gibt es drei potenzielle Wege der Wissensvermittlung:

- Professionalisierung (durch Lernen und Erfahrungsaustausch in Gruppen),

- Objektivierung (durch die physische Verankerung von Standards, z.B. in Hardware und Software) und

- organisatorische Verankerung (durch Methoden, Regeln, Standards, Routinen und Strukturen).

Mit Hilfe von vier Fallstudien zeigt er auf, welche Rolle die Kosten, das Innovationspotenzial und die Wissensvermittlung in großen IV-Projekten spielen. Die Interpretation zeigt auf, dass der Kauf von Standardsoftware den Zugriff auf objektiviertes Wissen beinhaltet und Kostenvorteile impliziert. Allerdings birgt der Zugriff auf objektiviertes Wissen die Gefahr der Abhängigkeit vom Softwarehersteller und kann zu erheblichen Schwierigkeiten (z.B. ex-post Kosten) führen, wenn die Software notwendige Funktionalitäten nicht abdeckt. Bei Projekten, in denen ein hoher Grad an spezifischem Unternehmenswissen notwendig ist, wird entweder die interne Entwicklung einer Individualsoftware oder die gemeinschaftliche Entwicklung einer spezifischen Standardsoftware in Kooperation mit einem Softwarehersteller gewählt. Bei letzterer Variante stellte der Austausch von komplementärem Wissen (Unternehmenswissen der Geschäftsprozesse versus technologisches Wissen des Externen) eine besondere Herausforderung dar. Um diesen Wissenstransfer zu ermöglichen, ist ein sozio-technologischer Zusammenschluss zu schaffen [Scar98, S. 156]. Dieser spiegelt die Vermittlung des Wissens im Sinne von Professionalisierung wider. Der Anreiz für den Softwarehersteller, das gewonnene geschäftsspezifische Wissen an andere Kunden in der gleichen 
Branche weiterzugeben, spielt für die Motivation zum Wissenstransfer eine wichtige Rolle (Anreize). [BeWa98] legen bei ihrer Fallstudienuntersuchung ebenfalls den Schwerpunkt auf die Rolle des Wissens beim IV-Outsourcing. Sie untersuchen, wie die im Rahmen der Softwareakquisition und der -implementierung auftretenden Herausforderungen des Wissensmanagements die Outsourcingentscheidung beeinflussen.

Dabei unterscheiden sie zwischen

- kodifiziertem Wissen in Artefakten (z.B. Softwarepaket und seine Dokumentation) und

- implizitem Wissen (,tacit knowledge“, in den Köpfen der Mitarbeiter).

Für die Evalierung des Outsourcing beurteilen sie beide Wissensarten vor dem Hintergrund der gegenwärtigen und erwünschten Geschäftsprozesse. Diese können individuell oder standardisiert sein. Ihre Fallstudien belegen, dass externe Dienstleister insbesondere implizites Wissen hinsichtlich der Software haben. Wissen über Standardprozesse können sie sich schnell aneignen. Bei individuellen Prozessen ist allerdings ein Lernprozess nötig. Als Anreiz für einen solchen Lernprozess kann die Weiterverwertung des Wissens bei anderen Kunden dienen. Problematisch wird es allerdings, wenn der externe Anbieter dauerhaft neues implizites Wissen zu generieren hat, das auf die Bedürfnisse von individuellen Geschäftsprozessen abgestimmt sein muss.

Die wesentlichen in der Literatur festgestellten Begründungszusammenhänge des IV-Outsourcing lassen sich in folgendem Strukturmodell (Abbildung 1) darstellen:

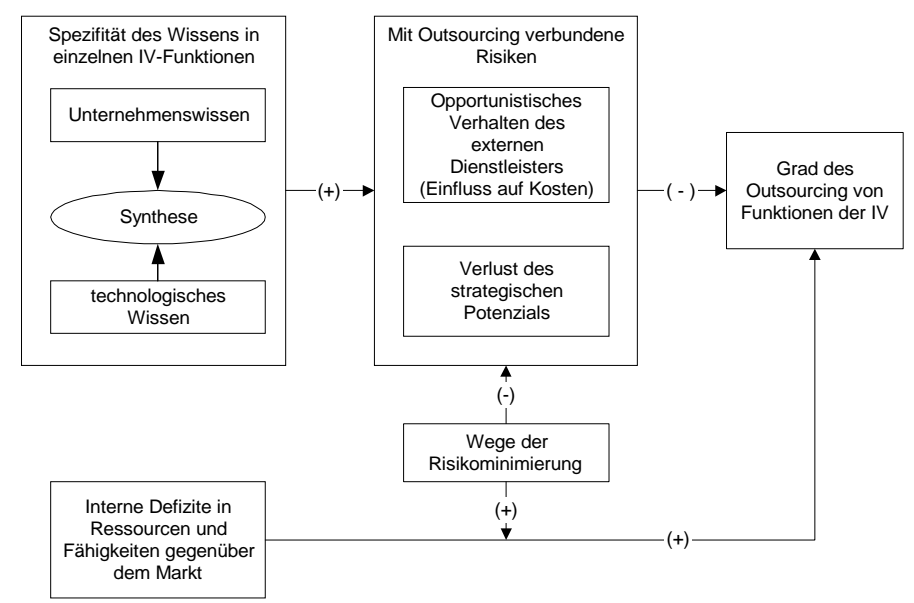

Abbildung 1: Strukturmodell des Einflusses von Wissen auf das IV-Outsourcing 


\section{Wissensorientierte Analyse der Aufgaben im ERP- Lebenszyklus}

Bevor die aus der Literatur herausgearbeiteten Begründungszusammenhänge auf die Outsourcingentscheidung bei ERP-Systemen bezogen werden können, sind zunächst die Aufgaben, die im Lebenszyklus von ERP-Systemen anfallen, zu ermitteln. Diese bilden die potenziellen Gegenstände des Outsourcing. Anschließend wird das im ERP-Lebenszyklus notwendige Wissen in Kategorien eingeteilt. Darauf aufbauend wird ein Bezugsrahmen aufgespannt, der die Zuordnung von einzelnen Aufgaben im Lebenszyklus von ERP-Systemen zu verschiedenen Wissenskategorien und internen oder externe Aufgabenträgern ermöglicht.

\subsection{Aufgaben im ERP-Lebenszyklus}

Der Anwendungs- bzw. Softwarelebenszyklus stellt den Prozess dar, wie eine Software entwickelt, genutzt und gewartet wird, bis sie letztendlich deinstalliert wird. Gewöhnlich wird der Softwarelebenszyklus durch eine Folge zeitlich begrenzter Phasen und darin auszuführender Aktivitäten beschrieben [Krcm97]. Die verschiedenen Modelle zur Beschreibung des Softwarelebenszyklus sind auf die Eigenerstellung von Software bezogen. Diese eignen sich allerdings nur bedingt zur Beschreibung des Softwarelebenszyklus in Unternehmen, die ein ERP-Paket einsetzen. Die Entscheidung für ein ERP-Paket hat einen eigenständigen Lebenszyklus zur Folge.

Die gewählte Phaseneinteilung der vorliegenden Arbeit lehnt sich insbesondere an die vier Phasen nach [MaTa00] an. Sie umfasst (1) die Akquisitions-, (2) die Implementierungs-, (3) die Stabilisierungs- sowie (4) die Betriebs- und Verbesserungs-Phase. Für die weitere Betrachtung sind besonders diejenigen Aufgaben im ERP-Lebenszyklus relevant, die im direkten Zusammenhang mit dem ERP-System bzw. mit den durch das ERP-System unterstützten Geschäftsprozessen stehen. Es lassen sich die in Tabelle 1 aufgeführten ERP-bezogenen Aufgaben pro Phase unterscheiden [Dave00; MaTa00].

Die mit dem ERP-System verbundenen Aufgaben werden häufig in technische versus funktionale (bzw. anwendungsseitige) Aufgaben unterteilt. Die technischen Aufgaben betreffen einerseits die IT-Infrastruktur, z.B. die Installation und Wartung der Server (inkl. Betriebssystem, Festplattenspeicher, etc.), der Arbeitsplatzrechner und des Netzwerks. Andererseits beinhalten sie die Aufgaben, die direkt mit dem Betrieb des ERP-Systems zusammenhängen, z.B. die Überwachung und Optimierung der Datenbank, auf der das ERP-System aufgebaut ist, die Speichernutzung, die Benutzerverwaltung sowie Drucker- und Spoolverwaltung. Letztere werden bei SAP R/3 auch als Basistätigkeiten bezeichnet. Die funktionalen Aufgaben beziehen sich auf die im ERP-System abgebildeten Geschäftsprozesse. Zu ihnen zählen insbesondere die Konfiguration des ERP-Pakets, mögliche Modifi- 
kationen, die Integrationstests des ERP-Systems und die Schulung der Endanwender sowie die Organisation des Roll-Outs. Oftmals werden die funktionalen Aufgaben entsprechend der einzelnen Module organisiert.

\begin{tabular}{|c|c|c|}
\hline & Phasen & Aufgaben \\
\hline 1) & $\begin{array}{l}\text { Akquisition } \\
\text { (mehrere } \\
\text { Monate) }\end{array}$ & $\begin{array}{l}\text { - } \quad \text { Erstellung eines Pflichtenheftes auf Basis einer Ist-Analyse } \\
\text { - } \quad \text { Auswahl eines ERP-Pakets }\end{array}$ \\
\hline 2) & $\begin{array}{l}\text { Implemen- } \\
\text { tierung } \\
\text { (6 Monate } \\
\text { bis } 3 \text { Jahre) }\end{array}$ & 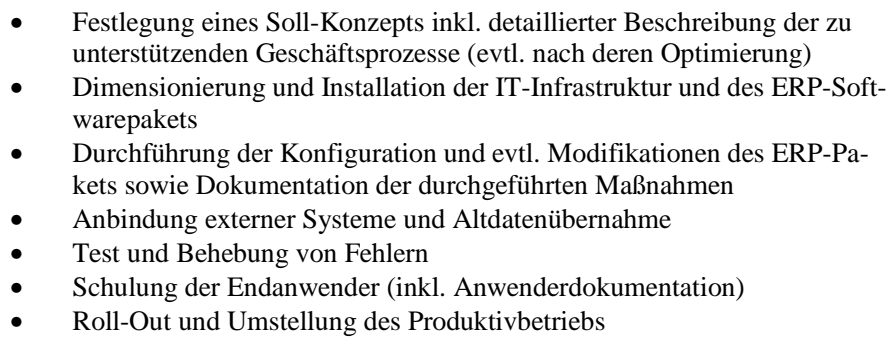 \\
\hline 3) & $\begin{array}{l}\text { Stabilisie- } \\
\text { rung } \\
\text { (bis } 6 \text { Mo- } \\
\text { nate) }\end{array}$ & $\begin{array}{ll}\text { - } & \text { Fehlerbehebung im ERP-System (bei Konfiguration oder Modifikationen) } \\
\text { oder der IT-Infrastruktur } \\
\text { - } & \text { Änderungen von Geschäftsabläufen } \\
\text { - } & \text { Verbesserung der Systemleistung (z.B. durch Erweiterung der Hardware) } \\
\text { - } & \text { Wiederholung der Schulungen oder zusätzliche Schulungen }\end{array}$ \\
\hline 4) & $\begin{array}{l}\text { Betrieb und } \\
\text { Verbesse- } \\
\text { rungen } \\
(10-15 \\
\text { Jahre })\end{array}$ & $\begin{array}{ll}\text { - } & \text { Durchführung von Releasewechseln } \\
\text { - } & \text { Unterstützung der Anwender \& Delta-Schulungen } \\
\text { - } & \text { Betrieb des ERP-Systems (IT-Infrastruktur und ERP-Basis) } \\
\text { - } & \text { Evtl. Kontinuierliche Prozessverbesserungen }\end{array}$ \\
\hline
\end{tabular}

Tabelle 1: Aufgaben im Lebenszyklus von ERP-Systemen

\subsection{Wissenskategorien im Lebenszyklus von ERP-Systemen}

Wissen entsteht durch die Kombination von Informationen mit der im Gedächtnis gespeicherten Erfahrung und äußert sich in Form von Kenntnissen und Fähigkeiten [Bea00]. Um die Erscheinungsformen des Wissens zu charakterisieren, wird in der Literatur eine Unterteilung in verschiedene Wissensarten vorgenommen. Als solche kann die Unterscheidung zwischen implizitem Wissen (,tacit knowledge“) und explizitem Wissen (,explicit knowledge“) eingestuft werden [NoTa95]. Implizites Wissen ist eng verwandt mit der ,human asset specificity“ in der Transaktionskostentheorie. Es kann zwischen notwendigen und hinreichenden Bedingungen unterschieden werden. Die intellektuellen Fähigkeiten der Mitarbeiter sind eine notwendige Bedingung. Ein hinreichendes Kriterium liefert die Frage, ob zur Verrichtung von Aufgaben Kenntnisse nötig sind, die nur durch einen „learning by doing“-Prozess, z.B. durch Teamarbeit im Unternehmen, erlangt werden können. 
Als Beispiel führt [Will81, S. 563] an: „Knowledge of a particular firm`s filing system (...) can be highly specific (nontransferable)." Explizites Wissen kann durch Symbole und durch Dokumentation „kodiert“ werden. Es ist leicht übertragbar, z.B. durch Schulungsmaßnahmen oder autodidaktisches Lernen.

Die Wissensarten können unterschiedlichen Aufgabenträgern zugeordnet werden. Das Wissen kann innerhalb der Unternehmung (intern) oder außerhalb der Unternehmung (extern), z.B. bei einem Berater, angesiedelt sein.

Um das zur Bewältigung der ERP-bezogenen Aufgaben benötigte Wissen analysieren zu können, ist es notwendig, dieses in Kategorien zu unterteilen. Als Ansatzpunkt für die vorliegende Arbeit werden die von [RoCh00, S. 631f.] mittels einer Literaturanalyse gewonnenen fünf Kategorien (1) „business knowledge“, (2) „technical knowledge“, (3) „product knowledge“, (4) „,company-specific knowledge“ und (5) „project knowledge“ herangezogen. Unter Berücksichtigung eigener Erkenntnisse aus über 25 persönlichen Interviews mit Unternehmensvertretern, die zu ihren Erfahrungen mit ERP-Systemen befragt wurden, propagieren die Autoren sieben verschiedene Wissenskategorien:

1. Best-Practice-Wissen: Diese Kategorie umfasst das Wissen über den bestmöglichen Ablauf von Geschäftsprozessen und der dazu notwendigen Organisationsstruktur. Als Beispiel sei ein nach den Prozeßkosten und Durchlaufzeiten optimierter Versandprozess genannt. Viele Beratungsgesellschaften geben an, dieses Wissen durch zahlreiche Business-Process-RengineeringProjekte in Unternehmen gesammelt, verfeinert und umgesetzt zu haben. Das Best-Practice-Wissen ist per Definition unternehmensunspezifisch und nicht an ein ERP-Paket gebunden. Es ist meist unternehmensextern und nur in impliziter Form vorhanden.

2. ERP-Funktionalitätswissen: Dieses Wissen betrifft zum einen die vielfältigen im ERP-Softwarepaket vorhandenen Funktionalitäten, welche die in der Software abgebildeten Referenzprozesse widerspiegeln. Zum anderen beinhaltet es, wie diese mittels der Konfiguration (= Parametereinstellungen) realisiert werden können. Diese Wissen ist hochgradig abhängig von dem eingesetzten ERP-Paket und zumindest zum Beginn des Lebenszyklus eines ERP-Systems meist nur extern vorhanden. Es ist zwar teilweise explizit in der Softwaredokumentation der ERP-Hersteller aufgeführt, die tatsächliche Anwendung erfordert wegen der Komplexität der ERP-Pakete allerdings einen Lernprozess.

3. Programmierwissen: Diese Kategorie beinhaltet das Wissen über die Programmiersprache des ERP-Pakets (z.B. ABAP/4 bei SAP R/3) und die Architektur der ERP-Software. Dieses Wissen ist notwendig, um Modifikationen an dem ERP-Paket vornehmen zu können. Es ist ebenfalls explizit vorhanden - muss aber erlernt werden. Es ist zumindest im Anfangsstadium des ERP-Lebenszyklus meist nur extern vorhanden. 
4. IT-Infrastruktur- und ERP-Basiswissen: Diese Kategorie beinhaltet technisches Wissen über die Hardware (z. B. Server, Arbeitsplatzrechner und Netzwerke inklusive der Systemsoftware) und über die Basisdienste des ERPPakets (u.a. Datenbank, ERP-Kernel, Nutzerverwaltung) essentiell. Dieses Wissen ist abhängig von der eingesetzten IT-Infrastruktur und dem ERP-Paket. Es ist bis auf gewisse „Kniffe“ explizit vorhanden und kann gut durch Schulungen erlernt werden.

5. Prozesswissen: Dieses Wissen umfasst die unternehmensspezifische Art und Weise der Durchführung der Geschäftsprozesse. Es ist oftmals an einzelne Personen oder Gruppen im Unternehmen geknüpft und beinhaltet einen hohen Grad an Erfahrungswissen. Es gilt insbesondere auch die Zusammenhänge über Abteilungsgrenzen hinweg zu kennen. Dieses Wissen ist meist nur implizit innerhalb der Unternehmensgrenzen vorhanden.

6. Legacy-System-Wissen: Dieses Wissen betrifft vor allem individuell entwickelte Anwendungen. Das Wissen über ihre Spezifikation ist meist an einzelne Personen gebunden und schlecht dokumentiert. Im Rahmen der ERPEinführung sind häufig Daten aus den Altsystemen zu übernehmen. Dieses Wissen existiert meist nur implizit.

7. Anwendungswissen: Diese Wissenskategorie stellt das Wissen über die korrekte Anwendung des ERP-Pakets dar. Sie ist essentiell, um in der täglichen Nutzung des ERP-Pakets die beabsichtigten Ergebnisse zu erzielen. Diese Wissenskategorie ist vom ERP-Paket und dem Unternehmen abhängig. Das Anwendungswissen wird den Endanwendern meist durch Schulungsmaßnahmen vermittelt und ist häufig in sog. Anwendungsdokumentationen explizit verfügbar.

\subsection{Bezugsrahmen des aufgaben- und aufgabenträger- spezifischen Wissens}

Um die Wahlsituation bei der Outsourcingentscheidung transparent zu machen, sind die alternativen Aufgabenträger den einzelnen Aufgaben und Wissenskategorien im ERP-Lebenszyklus gegenüberzustellen. Generell können unternehmensinterne und -externe Aufgabenträger unterschieden werden.

Als unternehmensinterne Aufgabenträger kommen zunächst die Mitarbeiter der IV-Abteilung in Frage. Diese sind in größeren Unternehmen in der Regel arbeitsteilig den einzelnen IV-Funktionen zugeordnet und können ihren Fähigkeiten entsprechend den Aufgaben im ERP-Lebenszyklus zugeordnet werden. Des Weiteren besteht die Möglichkeit, dass sich im Zuge einer organisatorischen Abschwungtendenz IV-Kompetenzen in die Fachabteilungen verlagert haben [Hein96]. Im ERP-Kontext spricht man in diesem Zusammenhang von „Keyusern“ bzw. „Su- 
perusern“ [Dave00, S. 186f.]. Diese sind von den „normalen“ Endanwendern abzugrenzen.

Die unternehmensexternen Aufgabenträger können in unterschiedliche Typen eingeteilt werden. In Anlehnung an die Ergebnisse einer umfangreichen Fallstudienuntersuchungen von [MiFi97] können fünf verschiedene IT-Dienstleistungsunternehmen unterschieden werden. Es handelt sich um (1) Berater, (2) Systemhäuser, (3) Hardwareanbieter, (4) ehemalige IV-Abteilungen und (5) generische Outsourcer. Als sechste Kategorie sollen (6) Freiberufler berücksichtigt werden.

In Abbildung 2 ist dargestellt, auf welche Art und Weise die Aufgaben den Wissenskategorien und den Aufgabenträgern zugeordnet werden können. Anhand der Aufgabe „Konfiguration“ ist beispielhaft eine mögliche Zuordnungsvariante dargestellt. Um die Parametrierung bei der Konfiguration durchzuführen ist sowohl Wissen um die Funktionalität der Software als auch der Geschäftsprozesse notwendig. In dem dargestellten Szenario wird davon ausgegangen, dass das Wissen asymmetrisch verteilt ist. Während das ERP-Funktionalitätswissen bei einem Berater liegt, ist das Prozesswissen bei den funktionalen Mitarbeitern der internen IV-Abteilung und den Keyusern angesiedelt.

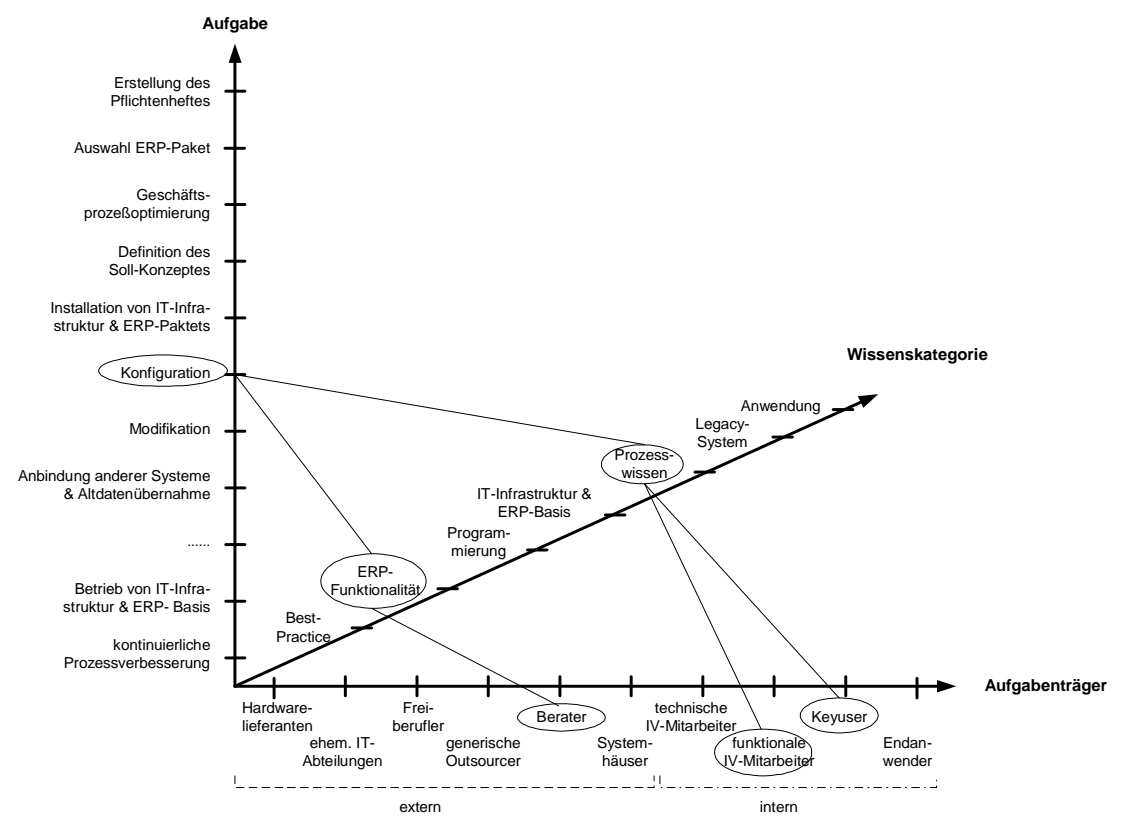

Abbildung 2: Bezugsrahmen des aufgaben- und aufgabenträgerspezifischen Wissens 


\section{Exemplarische Anwendung von Bezugsrahmen und Strukturmodell}

Das vorher genannte Beispiel läßt allerdings die Frage offen, welche Implikationen die dargestellte Wissensverteilung auf die Outsourcingentscheidung hat. Zur Beantwortung dieser Frage kann auf die Begründungszusammenhänge des in Abbildung 1 dargestellten Strukturmodells zurückgegriffen werden. Im Folgenden soll anhand des Beispiels,,Konfiguration“ die Anwendung skizziert werden. Es wird davon ausgegangen, dass im Zuge des Auswahlprozesses die Wahl auf ein bestimmtes ERP-Paket gefallen ist und dass interne Defizite im ERP-Funktionalitätswissen vorliegen. Das Strukturmodell besagt, dass bei Vorhandensein eigener Defizite ausgelagert wird, und dass diese Tendenz umso höher ausfällt, je eher es Wege der Minimierung des Outsourcingrisikos gibt. Es stellt sich demnach die Frage, welche Risiken im Rahmen der Konfiguration mit dem externen Bezug von ERP-Funktionalitätswissen verbunden sind. Hierzu ist zunächst die Spezifität der Aufgabe „Konfiguration“ zu beurteilen. Das ERP-Funktionalitätswissen per se kann nicht als spezifisch eingestuft werden. Es gilt allerdings zu berücksichtigen, dass die Konfiguration eine Synthese aus ERP-Funktionalitätswissen und Unternehmenswissen verlangt. Letzteres soll als unternehmensspezifisch eingestuft werden. Die Synthese verlangt daher einen Wissensaustausch zwischen internen IV-Mitarbeitern und Keyusern sowie externen Beratern. Hierbei tritt die Gefahr des opportunistischen Verhaltens des Externen auf. Das Verstehen der Prozesse und der Bedürfnisse des Kunden verlangt spezifische Investitionen des Externen. Er wird bestrebt sein, diese im Laufe der Vertragslaufzeit zu überkompensieren. Um dieses Risiko zu vermindern, können Anreize die Berater zum „freiwilligen“ Wissensaustausch bewegen. Der Idealfall tritt dann ein, wenn dieser den Wissensgewinn, z.B. spezifisches Branchenwissen, bei anderen Kunden weiterverwerten kann. Für ihn besteht in diesem Fall die Möglichkeit, den Kunden als Referenzkunden zu benennen. Man kann von einer ,win-win“-Situation sprechen, da der Kunde gleichsam die Möglichkeit erhält, das ERP-Funktionalitätswissen des Externen zu absorbieren (= Wissenstransfer) und somit vom Berater unabhängig zu sein. Als weiteres Szenario ist vorstellbar, dass der Externe die ehemals internen Mitarbeiter übernimmt. In diesem Fall gelten die gleichen Überlegungen wie oben angeführt - mit einer Ausnahme: der Kunde gerät in ein langfristiges Abhängigkeitsverhältnis vom Externen und verliert sein spezifisches Prozesswissen. Dieses Risiko könnte durch eine Kapitalbeteiligung am externen Anbieter (= Joint Venture) begrenzt werden. Gleichsam muss man sich aber darüber im Klaren sein, dass die ehemals internen IT-Mitarbeiter nun auch externe Kunden betreuen und eine Erosion des unternehmensspezifischen Prozesswissens nicht ausgeschlossen werden kann.

Das skizzierte Beispiel zeigt auf, dass eine Varianztheorie von hoher praktischer Relevanz sein kann, da sie dem Praktiker die Möglichkeit gibt, den eigenen Entscheidungsprozess zu systematisieren und kritisch zu reflektieren. 


\section{Literatur}

[AnCu97] Ang, S.; Cummings, L. L.: Strategic Response to Institutional Influences on Information Systems Outsourcing. In: Organization Science, 8(1997)3, S. 235-256.

[ApRi00] Appelrath, H.-J.; Ritter, J.: R/3-Einführung: Methoden und Werkzeuge. Springer, Berlin u.a. 2000.

[Barn91] Barney, J.: Firm Resources and Sustained Competitive Advantage. In: Journal of Management, 17(1991)1, S. 99-120.

[Bea00] Bea, F. X.: Wissensmanagement. In: WiSt(2000)7, S. 362-367.

[BeWa98] Beath, C. M.; Walker, G.: Outsourcing of Application Software: A Knowledge Management Perspective. 31st Hawaii International Conference on System Sciences, Hawaii, USA 1998, S. 666-674.

[BrHM01] Brehm, L.; Heinzl, A.; Markus, M. L.: Tailoring ERP Systems: A Spectrum of Choices and their Implications. 34th Hawaii International Conference on System Sciences, Hawaii, USA 2001, S. 1-9.

[Dave00] Davenport, T. H.: Mission Critical: Realizing the Promise of Enterprise Systems. Harvard Business School Press, Boston 2000.

[DiGH01] Dibbern, J.; Güttler, W.; Heinzl, A.: Die Theorie der Unternehmung als Erklärungsansatz für das selektive Outsourcing der Informationsverarbeitung. In: Zeitschrift für Betriebswirtschaft (ZfB), 6(2001).

[DiHe01] Dibbern, J.; Heinzl, A.: Outsourcing der Informationsverarbeitung im Mittelstand: Test eines multitheoretischen Kausalmodells. In: Wirtschaftsinformatik, erscheint in 2001.

[Dunc98] Duncan, N. B.: Beyond Opportunism: A Resource-based View of Outsourcing Risk. 31st Hawaii International Conference on System Sciences, Hawaii, USA 1998, S. 675-684.

[Ear196] Earl, M. J.: The risks of outsourcing IT. In: Sloan Management Review, 37(1996)3, S. 26-32.

Hein93] Heinzl, A.: Die Ausgliederung der betrieblichen Datenverarbeitung : eine empirische Analyse der Motive, Formen und Wirkungen. 2. Aufl., Poeschel, Stuttgart 1993.

[Hein96] Heinzl, A.: Die Evolution der betrieblichen DV-Abteilung: eine lebenszyklustheoretische Analyse. Physica, Heidelberg 1996.

[Krcm97] Krcmar, H.: Informationsmanagement. Springer, Berlin u. a. 1997.

[LaWF95] Lacity, M. C.; Willcocks, L. P.; Feeny, D. F.: IT Outsourcing: Maximize Flexibility and Control. In: Harvard Business Review, May-June(1995), S. 84-93.

[Loh94] Loh, L.: An Organizational-Economic Blueprint for Information Technology Outsourcing: Concepts and Evidence. 15th International Conference on Information Systems, Vancouver, Canada 1994, S. 73-89. 
[MaTa00] Markus, M. L.; Tanis, C.: The Enterprise Systems Experience - From Adoption to Success. In: R. W. Zmud (Hrsg.): Framing the Domains of IT Research: Glimpsing the Future Through the Past. Pinnaflex Educational Resources, Inc., Cincinnati, OH 2000, S. 173-207.

[MeKn98] Mertens, P. and Knolmayer, G.: Organisation der Informationsverarbeitung: Grundlagen - Aufbau - Arbeitsteilung, Wiesbaden 1998.

[MiFi97] Michell, V.; Fitzgerald, G.: The IT Outsourcing Market-Place: Vendors and their Selection. In: Journal of Information Technology, 12(1997), S. 223-237.

[Mohr82] Mohr, L. B.: Explaining Organizational Behavior: The Limits and Possibilities of Theory and Research. Jossey-Bass Publishers, San Francisco, Washington, London 1982 .

[NRRC96] Nam, K.; Rajagopalan, S.; Rao, H. R.; Chaudhury, A.: A Two-Level Investigation of Information Systems Outsourcing. In: Communications of the ACM, 39(1996)7, S. 36-44.

[NeRS96] Nelson, P.; Richmond, W.; Seidmann, A.: Two dimensions of software acquisition. In: Communications of the ACM, 39(1996)7, S. 29-35.

[NoTa95] Nonaka, I.; Takeuchi, H.: Die Organisation des Wissens. Campus, Frankfurt/Main u.a. 1995.

[PiMa92] Picot, A.; Maier, M.: Analyse- und Gestaltungskonzepte für das Outsourcing. In: Information Management, 4(1992), S. 14-27.

[PoZe98] Poppo, L.; Zenger, T.: Testing Alternative Theories of the Firm: Transaction Cost, Knowledge-Based, and Measurement Explanations for Make-or-Buy Decisions in Information Services. In: Strategic Management Journal, 19(1998), S. 853-877.

[QuHi94] Quinn, J. B.; Hilmer, F. G.: Strategic Outsourcing. In: Sloan Management Review, 35(1994)4, S. 43-55.

[RoCh00] Rosemann, M.; Chan, R.: Structuring and Modeling Knowledge in the Context of Enterprise Resource Planning. The Pacific Asia Conference on Information Systems, Hong Kong 2000, S. 623-640.

[Scar98] Scarbrough, H.: The External Acquisition of Information Systems Knowledge. In: L. P. Willcocks und M. C. Lacity (Hrsg.): Strategic Sourcing of Information Systems. John Wiley \& Sons Ltd, 1998, S. 137-161.

[TRDW94] Teece, D. J.; Rumelt, R. P.; Dosi, G.; Winter, S. G.: Understanding Corporate Coherence: Theory and Evidence. In: Journal of Economic Behavior and Organization, 23(1994), S. 1-30.

[TeCG95] Teng, J. T. C.; Cheon, M. J.; Grover, V.: Decisions to Outsource Information Systems Functions: Testing a Strategy-Theoretic Discrepancy Model. In: Decision Sciences, 26(1995)1, S. 75-103.

[Will81] Williamson, O. E.: The Economics of Organization: The Transaction Cost Approach. In: American Journal of Sociology, 87(1981)3, S. 548-577. 